\title{
Symbolic calculus on weighted group algebras
}

by

T. PYTLIK (Wroctaw)

Abstract. Let $G$ be a locally compact group of polynomial growth and $\omega$ a nonconstant polynomial weight on $G$. It is shown that $L^{1}(G, \omega)$ is a symmetric algebra and that functions with arbitrarily small support operate on hermitian $L^{2}$-functions in it.

Let $A$ be a semi-simple, commutative Banach *-algebra. The Gelfand transform ^ maps $A$ onto a subalgebra $\hat{A}$ in $C_{0}(M)$ of all continuous functions vanishing at infinity on the space $M$ of all maximal ideals of $A$. A function $F: R \rightarrow C$ with $F(0)=0$ operates on an element $f \in A$ if the range of $\hat{f}$ is real and $F \circ \hat{f} \in \hat{A}$.

In 1959 Helson, Kahane, Katznelson and Rudin [3] showed that if $F$ operates on every $f=f^{*}$ in $L^{1}(G)$, where $G$ is an infinite locally compact abelian group, then $F$ is real analytic. On the other hand, under some assumptions on a weight $\omega$ on a discrete abelian group $G$ also nonanalytic functions operate on $l^{1}(G, \omega)$ (cf. [2], [6], [7] and [8]).

All these results are obtained by estimating the growth of $\|u(n f)\|$ as $n \rightarrow \infty$, where $u(f)=\sum_{k=1}^{\infty} \frac{i^{k} f^{k}}{k !}$. In particular,

$$
\|u(n f)\|=O\left(e^{n^{\gamma}}\right) \quad \text { as } \quad n \rightarrow \infty
$$

for a $\gamma, 0<\gamma<1$, guarantees that functions with arbitrarily small supports operate on $f$.

J. Dixmier [1] extended the notion of function operating on an element $f$ in a non-commutative Banach *-algebra $B$ by repeating the above definition with $A$ being the maximal commutative Banach subalgebra of $B$ containing $f$. He proved that, if $G$ is a nilpotent group (or, generally, a group of polynomial growth), then any function $F$, where $F(0)=0$ and $F^{\prime}$ is differentiable sufficiently many times, operates on every $f$ in the algebra $L^{1}(G)$, provided that $f$ is an $L^{2}$-function with compact support. Later A. Hulanicki [4] obtained a result of this type for nilpotent groups of class 2 and hermitian functions in $L^{1}(G, \omega) \cap L^{2}(G)$, where $\omega$ is a polynomial (c.f. (2) below) non-constant weight on $G$ - 
In this note we investigate the algobra $L^{1}(G, \omega)$, whero $G$ is a locally compact group of polynomial growth and $\omega$ a polynomial woight with $\int_{G} \omega^{-p}(s) d s<+\infty$ for some $p>0$.

Theorem 1 is a generalization of our earlier result [9] and shows that $L^{1}(G, \omega)$ is symmetric. If $G$ is that of $[5]$, we get an example of a dense symmetric subalgebra in non-symmetric algebra $l^{1}(G)$.

In Theorem 2 we prove (1) for any hermitian $L^{2}$-function $f$ in the algebra $L^{1}(G, \omega)$. The condition $f \in L^{2}\left(G^{4}\right)$ is of eourse dutomatically satisfied if $G$ is discrete; thus Theorem 2 implies that somes non-analytic classes of functions operate on the algebra $l^{1}(G, \omega)$.

Let $G$ be a locally compact topological group. $\Lambda$ mousurable and locally bounded (e.g. bounded on compact sots) function $a:(x \rightarrow[1, \infty)$ is called a weight if $\omega(u s) \leqslant \omega(u) \omega(s)$ and $\omega\left(s^{-1}\right)=\omega(s)$ for ovory $u, s \in G$. The space $L^{1}(G, \omega)$ of all complex functions $f$ on $G$ for which $f \cdot \omega$ bolongs to $L^{1}(G)$ is a Banach *algebra with the algobra oporations inhorited. from $L^{1}(G)$ and the norm

$$
\|f\|_{\omega}=\|f \cdot \omega\|_{1} \text {. }
$$

It is obvious that the sum and the product of two weights is a woight and the same is true for any positive power of a woight. Wo say that a weight $\omega_{1}$ is dominated by $\omega_{2}$ if there is a positive constant $M$ such that $\omega_{1} \leqslant M \omega_{2}$. Two weights $\omega_{1}, \omega_{2}$ are equivalent if $\omega_{1}$ is dominated by $\omega_{2}$ and $\omega_{2}$ is dominated by $\omega_{1}$. For equivalent weights $\omega_{1}, \omega_{2}$ the algebras $L^{1}\left(G, \omega_{1}\right)$ and $L^{1}\left(G, \omega_{2}\right)$ are isomorphic. Wo call $\omega_{1}$ a polynomial weight if

$$
\frac{\omega(u s)}{\omega(u)+\omega(s)} \text { is bounded on } G \times G \text {. }
$$

One can easily verify that the sum of two polynomial woights and any positive power of polynomial weight are polynomial woights. That this is not true for the product we will see in Example 3.

ExAMPLe 1. Let $G_{1} \subset G_{2} \subset \ldots$ bo an increasing sequeneo of closed subgroups of a locally compact group $G$ such that $\bigcup_{n+1}^{\infty} a_{n} \ldots a$. Lot $a_{1}$ $\leqslant \alpha_{2} \leqslant \ldots$ be an arbitrary increasing sequences of non-nogati've numbers. Then the function $\omega: G \rightarrow[1, \infty)$ definod by

$$
\omega=1+\sum_{n=1}^{\infty} \alpha_{n} 1_{\alpha_{n+1} \ldots c_{n}}
$$

is a weight on $G$ if it is locally bounded (for oxample if any compact set of $G$ is fully contained in some $G_{n}$ ). It satisfies the oquality

and therefore it is a polynomial weight.
ExAMPLe 2. Let $G$ be a compactly generated group. Let $K$ be a precompact symmetric (e.g. $K^{-1}=K$ ) open set in $G$ such that $\bigcup_{n=1}^{\infty} K^{n}=G$. Define $\sigma_{K}(s)=\inf \left\{n: s \in K^{n}\right\}$. Then $\sigma_{K}(s u) \leqslant \sigma_{K}(s)+\sigma_{K}(u)$ for all $s, u \in G$ and hence

$$
\begin{gathered}
\Omega_{K}(s)=e^{\sigma} K^{(s)}, \quad s \in G, \\
\omega_{K}(s)=1+\sigma_{K}(s), \quad s \in G
\end{gathered}
$$

are weights on $G$. If the group $G$ is non-compact (in this case $\sigma_{K}$ is unbounded on $G$ ) then only the second weight is polynomial.

Proposixion 1. Let $G$ be a compactly generated group. Let $K$ and $\sigma_{K}$ be as in Example 2. Every weight $\omega$ on $G$ is dominated by $\Omega_{K}^{\delta}$ for suitable $\delta>0$. If $\omega$ is a polynomial weight then it is dominated by $\omega_{K}^{\delta}$.

Proof. Denote $A=\sup _{s \in K} \omega(s)<+\infty$. If $s \in K^{n}-K^{n-1}$, then

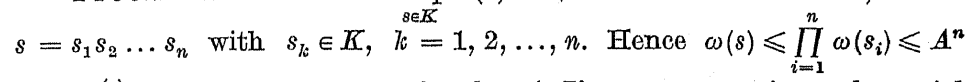
$=A^{\sigma_{K}(s)}$ and it suffices to put $\delta=\log _{e} A$. If, moreover, $\omega$ is a polynomial weight and $C$ is a bound of (2) then for $n=2^{m}$ we have $\omega(s) \leqslant A(2 C)^{m}$ $=A n^{\delta} \leqslant A \omega_{K}^{s}(s)$, where $\delta=1+\log _{2} C$. For if $2^{m}<n<2^{m+1}$ then $s$ $=s_{1} s_{2}$, where $s_{1}, s_{2} \in K^{2^{m}} ;$ thus $\omega(s) \leqslant \omega\left(s_{1}\right) \omega\left(s_{2}\right) \leqslant A^{2} \omega_{K}^{\delta}\left(s_{1}\right) \omega_{K}^{\delta}\left(s_{2}\right)$ $\leqslant A^{2} \omega_{K}^{28}(s)$.

EXAMPLE 3 . On the additive group $R^{2}$ the functions $\omega_{1}, \omega_{2}$ given by $\omega_{i}\left(\left(x_{1}, x_{2}\right)\right)=1+\left|x_{i}\right|, i=1,2$, are polynomial weights, but their product $\omega=\omega_{1} \omega_{2}$ is not. Indeed,

$$
\frac{\omega((t, t))}{\omega((t, 0))+\omega((0, t))}=\frac{1+|t|}{2}, \quad t \in R
$$

is unbounded.

LEMma 1. Let $\omega$ be a polynomial weight. There exists a constant $\theta$ such that for every $f, g$ in $L^{1}(G, \omega)$ we have

$$
\|f * g\|_{\omega} \leqslant O\left(\|f\|_{\omega}\|g\|_{1}+\|f\|_{1}\|g\|_{\omega}\right) .
$$

This is a trivial consequence of (2).

ColrollaARY 1. Let $\omega$ be a polynomial weight and let $f \in L^{1}(G, \omega)$. The spectral radii $r_{\omega}(f)$ and $r(f)$ in algebras $L^{1}(G, \omega)$ and $L^{1}(G)$ coincide. Proof. By (5) we have

$$
\left\|f^{2}\right\|_{\omega} \leqslant 2 O\|f\|_{\omega}\|f\|_{1},
$$

$$
\left\|f^{4}\right\|_{\omega} \leqslant 2 C\left\|f^{2}\right\|_{\omega}\left\|f^{2}\right\|_{1} \leqslant(2 C)^{2}\|f\|_{\omega}\|f\|_{1}^{3},
$$

and so on. Generally

$$
\left\|f^{2^{n}}\right\|_{\omega} \leqslant(2 C)^{n}\|f\|_{\omega}\|f\|_{1}^{2^{n}-1}
$$


Therefore

$$
r_{\omega}(f)=\lim _{n \rightarrow \infty}\left\|f^{2^{n}}\right\|_{\omega}^{2-n} \leqslant\|f\|_{1} .
$$

Substituting $f^{n}$ instead of $f$ in the last inequality, wo get

$$
r_{\omega}(f)=\left[r_{\omega}\left(f^{n}\right)\right]^{1 / n} \leqslant\left\|f^{n}\right\|_{1}^{1 / n},
$$

and thus $r_{\omega}(f) \leqslant r(f)$. The converso inequality is obvious, and so $r_{\omega}(f)$ $=r(f)$ and the corollary follows.

Corollary 2. Let $\omega$ be a polynomial weight. Every oontinuous homomorphism of $L^{1}(G, \omega)$ into a commutative $C^{*}$ algebra can be extended to the whole $L^{1}(G)$.

Proof. Let $A$ be a commutative $O^{*}$ algebra and lot $\varphi: L^{1}(G, \omega) \rightarrow A$. For $f \in L^{1}(G, \omega)$ we have

$$
\|\varphi(f)\|=\lim _{n \rightarrow \infty}\left\|\varphi\left(f^{n}\right)\right\|^{1 / n}=r_{\omega}(f): r(f) \leqslant\|f\|_{\perp} .
$$

So $\varphi$ is continuous in the $L^{1}(G)$ norm.

Remark 1 . The results of Corollaries 1 and 2 are not true for erery weight. This is shown in the following:

ExAMPLE 4. Let $G$ be the additive group of real numbers and $\omega(t)$ $=e^{|l|}$. The mapping $f \rightarrow \hat{f}(z)=\int_{-\infty}^{+\infty} f(t) e^{-i z t} d t$ is a continuous linoar and multiplicative functional on $L^{-\infty}(G, \omega)$ for any complex number $\approx$ from the set

Therefore

$$
D=\{z \in \theta:-1 \leqslant \operatorname{Im} z \leqslant 1\} \text {. }
$$

$$
r_{\omega}(f) \geqslant \sup _{z \in D}|\hat{f}(z)| .
$$

In particular, for the characteristic function of the interval $(0,1)$ wo have $\left\|f^{n}\right\|_{1}=\|f\|_{1}^{n}=1$; thus $r(f)=1$, but

$$
\hat{f}(i)=\int_{0}^{1} e^{t} d t=e-1
$$

and so $r_{\omega}(f) \geqslant e-1>r(f)$.

Lwimara 2. Let $\omega$ be a polynomial woight on $a^{2}$ sush that $\omega^{-m}$ bolongs to $L^{p}(G)$ for a $0<p<\infty$. Suppose $f \in L^{1}(G, \omega) \cap L^{2}(G)$. Then there exists a constant $A$, depending only on $\omega$ and suoh that

$$
\|f\|_{1} \leqslant A\|f\|_{2}^{2 /(p+2)} \cdot\|f\|_{\omega}^{p /(p)+2 \cdot 2)} .
$$

Proof. Let $\alpha$ be an arbitrary positivo number and lot

$$
U_{\alpha}=\{x \in G: \omega(x) \leqslant \alpha\}
$$

Then, since $1 \leqslant \alpha^{p} \omega^{-p}(x)$ for $x \in U_{\alpha}$, we have

$$
\left|U_{\alpha}\right|=\int_{U_{\alpha}} 1 d x \leqslant \alpha^{p} \int_{U_{\alpha}} \omega^{-p}(x) d x \leqslant B \alpha^{p}
$$

(here $\left|U_{\alpha}\right|$ denotes the Haar measure of the set $U_{a}$ ). Thus

$$
\begin{aligned}
\|f\|_{1}=\left\|f \cdot 1_{U_{\alpha}}\right\|_{1}+\left\|f \cdot 1_{G-U_{\alpha}}\right\|_{1} & \leqslant\|f\|_{2} \cdot\left\|1_{U_{\alpha}}\right\|_{2}+\left\|(f \cdot \omega) \cdot\left(1_{G-U_{\alpha}} / \omega\right)\right\|_{1} \\
& \leqslant\|f\|_{2} B^{1 / 2} \cdot \alpha^{p / 2}+\|f \cdot \omega\|_{1} \cdot\left\|\mathbf{1}_{G-U_{a}} / \omega\right\|_{\infty} \\
& \leqslant\|f\|_{2} B^{1 / 2} \cdot \alpha^{p / 2}+\|f\|_{\omega} \cdot \alpha^{-1} .
\end{aligned}
$$

For $\alpha=\left(\|f\|_{\omega}\|f\|_{2}^{-1} B^{-1 / 2}\right)^{2 /(p+2)}$ both factors of the right side of the last inequality are equal and so

$$
\|f\|_{1} \leqslant 2\left(\|f\|_{2} B^{1 / 2}\right)^{2 /(p+2)} \cdot\|f\|_{\omega}^{p /(p+2)} .
$$

This lemma has many consequences, so at first let us consider when the assumption on $\omega$ may bo realized.

Proposition 2. Let $G$ be a compactly generated locally compact group. There exists a polynomial weight $\omega$ on $G$ such that $\omega^{-1}$ belongs to $L^{1}(G)$ (or any $L^{p}(G), 1 \leqslant p<\infty$ ) if and only if $G$ is of polynomial growth, e.g. for any compact set $K \subset G$ there exists a polynomial $P$ such that $\left|K^{n}\right| \leqslant P(n)$, $n=1,2, \ldots$

Proof. Suppose $\omega^{-1}$ is integrable on $G$. Let $K$ be any compact set in $G$. It is contained in a compact set $K_{0}$ such that $K_{0}^{-1}=K_{0}$ and $\bigcup_{n=1}^{\infty} K_{0}^{n}$ $=G$. Then by Proposition 1, $\omega \leqslant \theta \omega_{K_{0}}^{8}$, and thus

$$
\left|K^{n}\right| \cdot(1+n)^{-\delta} \leqslant \int_{K_{0}^{n}}(1+n)^{-\delta} \leqslant \int_{\mathcal{K}_{0}^{n}} \omega_{\mathcal{K}_{0}^{-\delta}} \leqslant C \cdot \int_{G} \omega^{-1}=\theta_{1} .
$$

Therefore $\left|K^{n}\right| \leqslant O_{1}(1+n)^{\delta}$, and $G$ is of polynomial growth.

Conversely, if for a compact set $K$ in $G$ such that $K^{-1}=K, \bigcup^{\infty} K^{n}=G$ we have $\left|K^{n}\right| \leqslant C n^{k}, n=1,2, \ldots$, then $\omega_{K}^{-k-2}$ is integrable on $G$.

Now we are ready to prove a result which gives a generalization of [9], Theorem 6 .

THEOREM 1. Let $G$ be a locally compact group. If $\omega$ is a polynomial weight on $G$ such that $\omega^{-1}$ belongs to $L^{p}(G)$ for $a 0<p<\infty$, then the al gebra $L^{\mathrm{x}}(G, \omega)$ is symmetrio.

Proof. It suffices to prove $r_{\omega}(f) \leqslant \lambda(f)$ for any hermitian $f \in L^{1}(G, \omega)$ where $\lambda(f)$ denotes the norm of the operator $T_{f}$ acting on $L^{2}(G)$ by convolution.

Let $h$ be a function on $G$ such that $h \in L^{1}(G, \omega) \cap L^{2}(G)$. Then each function $f^{n} * h, n=1,2, \ldots$, satisfies the assumption of Lemma 2 , and so

$$
\left\|f^{n} * h\right\|_{1} \leqslant A\left\|f^{n} * h\right\|_{2}^{2 /(p+2)} \cdot\left\|f^{n} * h\right\|_{\omega}^{p /(p+2)}
$$


But $\left\|f^{n} * h\right\|_{2} \leqslant[\lambda(f)]^{n} \cdot\|h\|_{2}$ and $\left\|f^{n} * h\right\|_{\omega} \leqslant\left\|f^{n}\right\|_{\omega}\|h\|_{\omega} ;$ thus

$$
\varlimsup_{n \rightarrow \infty}\left\|f^{n} * h\right\|_{1}^{1 / n} \leqslant[\lambda(f)]^{2 /(p+2)} \cdot\left[r_{\omega}(f)\right]^{p /(p+2)} .
$$

By [9] Lemma 4, for a given $f$ there exists a continuous function $h$ with compact support such that $r(f) \leqslant \varlimsup_{n \rightarrow \infty}\left\|f^{n} * h\right\|_{1}$. Therefore by Corollary 1

$$
r(f) \leqslant[\lambda(f)]^{2 /(p+2)}[r(f)]^{p /(p+2)},
$$

and so $r(f) \leqslant \lambda(f)$.

Remark 2. It is shown in [5] that there exists a countable locally finite group $G$, e.g. every finitely generated subgroup of $G$ is tinito and such that $l^{1}(G)$ is not symmetric. It is easy to construct a weight $\omega$ on $G$ as in Example 1 such that $\omega^{-1}$ is integrable. Then by Theorem 1 the algebra $l^{1}(G, \omega)$ is a dense symmetric subalgebra in the non-symmetric algebra $l^{1}(G)$.

LeMria 3. Let $1<\lambda<2$. If $\alpha_{1}, a_{2}, \ldots$ is a sequenee of non-negative real numbers such that

(a) $\alpha_{n+m} \leqslant a_{n} \alpha_{m}, n, m=1,2, \ldots$,

(b) $a_{2 n} \leqslant n a_{n}^{\lambda}, n=1,2, \ldots$

then $\alpha_{n}=o\left(e^{n^{\gamma}}\right)$ for every $\gamma>\log _{2} \lambda$.

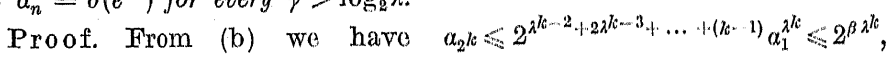
$k=0,1,2, \ldots$, where $\beta=(\lambda-1)^{-2}+\log _{2} a_{1}$. Now an arbitrary natural number $n$ is of the form $n=\varepsilon_{0} 2^{0}+\varepsilon_{1} 2^{1}+\ldots+\varepsilon_{m} 2^{m}$, where $\varepsilon_{k}$ is 0 or 1 , $\varepsilon_{m}=1$ and $\log _{2} n \leqslant m<1+\log _{2} n$. Therefore by (u)

$$
\alpha_{n} \leqslant \prod_{k=0}^{m} a_{2}^{e_{k}} \leqslant \prod_{k=0}^{m} 2^{\beta \lambda^{k}} \leqslant 2^{m \beta \lambda^{m}} \leqslant 2^{\left(1+\log _{2} n\right) \beta n^{\gamma_{0}}},
$$

where $\gamma_{0}=\log _{2} \lambda$. It is obvious that the left side of the last inequality is $o\left(e^{n^{\gamma}}\right)$ for every $\gamma>\gamma_{0}$.

Lemma 4. Let $\omega$ be as in Theorem 1. I'or $f \in L^{1}(G, \omega)$ let us define

$$
u(f)=\sum_{k=0=1}^{\infty} \frac{i^{r k} \cdot f^{\prime}}{k !} .
$$

If $f$ is hermitian and belongs to $L^{1}(G, \omega) \cap L^{2}(Q)$, then

$$
\begin{gathered}
\left\|f^{n}\right\|_{\omega}=[\lambda(f)]^{n} o\left(e^{n^{\nu}}\right), \\
\|u(n f)\|_{\omega}=o\left(e^{n^{\nu}}\right)
\end{gathered}
$$

for every $\gamma>\log _{2}((2 p+2) /(p+2))$.
Proof of (7). Ignoring the trivial case of $f=0$, we may assume $\lambda(f)=1$. We will show that the sequence $\alpha_{n}=D \cdot\left\|f^{n}\right\|_{\omega}, n=1,2, \ldots$ satisfies conditions (a) and. (b) of Lemma 3 for $\lambda=(2 p+2) /(p+2)$ and a suitable constant $D \geqslant 1$.

We have $\left\|f^{n+m}\right\|_{\omega} \leqslant\left\|f^{n}\right\|_{\omega}\left\|f^{m}\right\|_{\omega}$; thus condition (a) is satisfied. Now by (5) and (6)

$$
\left\|f^{2 n}\right\|_{\omega} \leqslant 2 O\left\|f^{n}\right\|_{1} \cdot\left\|f^{n}\right\|_{\omega} \leqslant 2 \sigma A\left\|f^{n}\right\|_{2}^{2 /(p+2)}\left\|f^{n}\right\|_{\omega}^{\lambda} .
$$

Since $\lambda(f)=1$, we have $\left\|f^{n}\right\|_{2} \leqslant\|f\|_{2}$ for each $n$; thus choosing $D$ such that $D^{\lambda-1} \geqslant 2 O A\|f\|_{2}^{2 /(p+2)}$ we get $(b)$.

Proof of (8). First observe that

$$
\|u(f)\|_{2} \leqslant\|f\|_{2} .
$$

Indeed, let $v$ be the analytic function defined by

$$
v(z)=\frac{e^{i z}-1}{z}=\sum_{k=1}^{\infty} \frac{i^{k} z^{k-1}}{k !}, \quad z \in C .
$$

Then we have $u(f)=v\left(T_{f}\right)(f)$, where $v\left(T_{f}\right)$ is the operator on $L^{2}(G)$ obtained by the action of $v$ on $T_{f}$. Therefore

$$
\begin{aligned}
\|u(f)\|_{2} & =\left\|v\left(T_{f}\right)(f)\right\|_{2} \leqslant\left\|v\left(T_{f}\right)\right\|\|f\|_{2} \\
& \leqslant \sup _{z \in S \mathrm{Sp} T}|v(z)|\|f\|_{2} \leqslant \sup _{t \in R}|v(t)|\|f\|_{2} \leqslant\|f\|_{2} .
\end{aligned}
$$

To prove (8) we will apply Lemma 3 with the sequence $a_{n}=$ $D\left(\|u(n f)\|_{\omega}+1\right)$ for a suitable constant $D \geqslant 1$. We have

$$
\begin{aligned}
\|u((n+m) f)\|_{\omega}+1 & =\|u(n f) * u(m f)+u(n f)+u(m f)\|_{\omega}+1 \\
& \leqslant\left(\|u(n f)\|_{\omega}+1\right)\left(\|u(m f)\|_{\omega}+1\right) ;
\end{aligned}
$$

thus (a) is satisfied. To prove (b), let us consider two cases: First, let $\|u(n f)\|_{\omega} \leqslant 1$. Then

$$
\|u(2 n f)\|_{\omega}+1 \leqslant\left(\|u(n f)\|_{\omega}+1\right)^{2} \leqslant 4 \leqslant 4\left(\|u(n f)\|_{\omega}+1\right)^{2},
$$

and so we have (b) for $D^{\lambda-1} \geqslant 4$. Second, let $\|u(n f)\|_{\omega} \geqslant 1$. Then by (5), (6) and (9)

$$
\begin{aligned}
\|u(2 n f)\|_{\omega}+1 & \leqslant\left(2 O\|u(n f)\|_{1}+2\right)\|u(n f)\|_{\omega}+1 \\
& \leqslant\left(20 A\|n f\|_{2}^{2 /(p+2)}\|u(n f)\|_{\omega}^{\prime /(p+2)}+2\right)\|u(n f)\|_{\omega}+1 \\
& \leqslant n\left(20 A\|f\|_{2}^{2 /(p+2)}+3\right)\|u(n f)\|_{\omega}^{\lambda},
\end{aligned}
$$

and it suffices to put $D^{\lambda-1} \geqslant 20 A\|f\|_{2}^{2 /(p+2)}+3$.

THEOREM 2. Let $G$ be a locally compact group. If $\omega$ is a polynomial weight on $G$ such that $\omega^{-1}$ belongs to $L^{p}(G)$ for $a 0<p<\infty$, then functions 
with arbitrarily small support operate on each hermitian $L^{2}$-function in the algebra $L^{1}(G, \omega)$.

Proof. This is an immediate consequence of (8).

\section{References}

[1] J. Dixmier, Opérateurs de rang fini dans les représentations unitaires, Inst. Hautes Études Sci. Publ. Math. 6 (1960), 13-25.

[2] Y. Domar, Harmonio analysis based on certain commutative Banach algebras, Acta Math. 96 (1956), 1-66.

[3] H. Helson, J.-P. Kahane, Y. Katznelson, W. Rudin, The funotions which operate on Fourier transforms, ibid. 102 (1959), $135-157$.

[4] A. Hulanicki, Growth of the $I^{1}$ norm of the oonvolution powers of functions on nilpotent groups of olass 2, Instituto Nazionale di Alta Matomatica, Symposia Mathematica 22 (1977), 439-447.

[5] - Invariant subsets of non-synthesis. Teptin algebras and non-symmetry, to appoar.

[6] M. Y. Katznolson, Sur le calcul symbolique dans quelques algèbres de Banach, Ann. Sc. École Norm. Sup. 63 (1959), 83-123.

[7] N. Leblanc, Calcul symbotique dans certains algèbres de sóries de frourier à poids, C. R. Acad. Sci. Paris 266 (1968), 339-341.

[8] N. B. Levina, Functions that operate on a group algebra wilh weight (in Russian), Teor. Funkcǐ̌ Funkcional. Anal. i Priložon. 25 (1976), 82-94.

[9] T. Pytlik, On the spectral radius of elements in group algebras, IBull. Aoad. Polon. Sci. 21 (1973), 899-902.

INSTITUTE OF MATHEMATXOS

UNIVERSITY OF WROCLAW

50-384 Wroclaw, Poland.

pl. Grunwaldzki 2/4 